\title{
Salud mental como efecto del desarrollo psicoafectivo en la infancia
}

\section{(1)Psicogente ISSN 0124-0137 EISNN 2027-212X}

\section{Mental health as an effect on childhood emotional development}

\author{
Viana Ángela Bustos Arcón , Ana Rita Russo de Sánchez \\ Universidad del Norte, Barranquilla, Colombia
}

Resumen

Objetivo: Comprender cómo el concepto de salud mental se relaciona con las nociones de interferencias en el desarrollo y recuperación psicoafectiva

Método: El presente artículo tiene como método la revisión documental. Es un estudio sistemático, metódico y organizado de textos, artículos y documentos sobre el tema estableciendo un análisis del concepto de salud mental como efecto del desarrollo psicoafectivo a la luz de la teoría psicodinámica, y las categorías analíticas corresponden al concepto de salud mental, interferencias en el desarrollo y recuperación psicoafectiva.

Resultados: La salud mental en la infancia y adolescencia es una preocupación reciente, pues se ha incrementado la incidencia de trastornos mentales en esta población. Las interferencias en el desarrollo se constituyen como dificultades en el curso esperado del desarrollo infantil repercutiendo en la salud mental. En Colombia, niños, niñas y adolescentes se ven expuestos a problemáticas que alteran el desarrollo psicoafectivo e impiden el despliegue de las potencialidades humanas y la consolidación de la personalidad.

Conclusiones: Se comprende que la salud mental es el efecto de los procesos de integración madurativo-constitucionales, mientras que las interferencias en el desarrollo como: abuso, maltrato, negligencia, abandono, entre otras, representan alteraciones en dicho proceso, pues afectan las condiciones biopsicosociales de los niños y niñas, por lo que la recuperación psicoafectiva se propone como una posibilidad para reestablecer la salud emocional y el balance óptimo indispensable para la superación de la adversidad y el curso esperado del desarrollo.

Palabras Claves:

Desarrollo psicoafectivo, Recuperación psicoafectiva, Interferencias en el desarrollo, Salud mental, Infancia.

Artículo de Investigación

Copyright 2018 by Psicogente

\section{Correspondencia de autores:} vianab71@gmail.com bustosv@uninorte.edu.co arusso@uninorte.edu.co

Recibido: $23-03-17$

Aceptado: $13-06-17$

Publicado: 01-01-18
Abstract

Objective: To understand the relationship between mental health, notions of development interference and emotional development.

Method: his paper has a documentary research method. It is based on a systematic, methodic and organized study of texts, articles and documents about the subject through a psychodynamic theory, it was established an analysis of the concept of mental health as an effect of emotional development and analytic categories, development interference and emotional recovery.

Results: Because of the statistics of mental disorders increasing on infancy and adolescences, mental health is a recent worry. The development interferences as difficulties on normal child development are associated to the loss of mental health during infancy. In Colombia, children and adolescences are exposed to problems which affect their emotional development and block the deployment of human potentialities and the consolidation of personality.

Conclusion: As a result, mental health is then known as the result of the maturating constitutional process, meanwhile the development interferences such as: abuse, mistreatment, negligence, neglect, among others, represent alterations in this maturating-constitutional process because all of them affects the child bio-psychosocial conditions, also according to these damages the emotional recovery turn into a proposal to restore the emotional health and the optimal balance necessary to overcome the adversity and promote the normal development on infancy.

Key words:

Emotional development, Mental health, Emotional development, Development interferences, Childhood.

Como citar este artículo (APA):

Bustos, V. \& Russo, A. (2018). Salud mental como efecto del desarrollo psicoafectivo en la infancia. Psicogente, 21(39), 183-202. http://doi.org/10.17081/psico.21.39.2830 


\section{INTRODUCCIÓN}

El estudio del desarrollo humano ha llevado a comprender la infancia como el momento evolutivo más fundamental del ciclo vital (Campo, 2014), pues es allí donde ocurren los procesos madurativoconstitucionales (Greenspan, 1992) más importantes de los individuos. Mass (2014) afirma que "El análisis de los fenómenos humanos, desde el campo de la subjetividad, ha permitido reconocer el sentido de los trazos históricos particulares del sujeto, así como los modos en que este se inserta en la cultura" (p.156). En este sentido, la infancia ocupa un lugar privilegiado en el desarrollo de las sociedades, a nivel político, económico y en materia de protección y cuidado de sus derechos, a través de leyes, decretos y resoluciones que garantizan sus derechos.

La salud mental en la infancia es una problemática de interés nacional e internacional, pues el reciente incremento de la incidencia de trastornos mentales convoca a pensar las condiciones actuales bajo las que se desarrollan los niños y niñas. En el mundo “(...) los datos epidemiológicos hasta el 2005 sugerían una prevalencia de trastornos mentales hasta del $20 \%$ de los niños y adolescentes; de estos, entre un $4 \%$ y un $6 \%$ requieren atención en servicios de salud" (Minsalud \& Colciencias, 2015, p.206). Mientras que, de acuerdo con la Encuesta Nacional en Salud Mental en Colombia se advierte que entre las problemáticas mentales "Para la población infantil de 7 a 11 años se incluyen: depresión mayor, ansiedad de separación, trastorno de ansiedad generalizada, trastorno negativista desafiante, trastorno por déficit de atención e hiperactividad (TDAH) y trastorno de la conducta" (Minsalud \& Colciencias, 2015, p.206). Lo anterior representa un compromiso por garantizar la salud mental óptima en la infancia.

En la actualidad la infancia se ve más comprometida en sus posibilidades debido a las condiciones económicas, políticas, culturales, entre otras, de la sociedad postmoderna. "Bajo este panorama, la situación de amenaza de la infancia a nivel mundial es alarmante (...)" (Martínez, Robles, Utria \& Amar, 2014, p.135). Los esfuerzos se observan minúsculos cuando las cifras nacionales e internacionales en materia de infancia ocupan preocupaciones y sobresaltos frente a las atroces dificultades que ocurren en la infancia, desde falta de servicios básicos hasta abusos o maltratos, por mencionar algunas. Cada día, los canales populares (televisión, radio, internet, etc.) se invaden de noticias que dejan perplejo al más desprevenido sobre el peligro que implica ser niño/a hoy día, la ausencia de los padres, o el empoderamiento desbocado del niño ha dejado sin efecto a los adultos frente a los momentos evolutivos y la crianza. Bien podrían ser pensados los trastornos mentales en la infancia como un síntoma de las sociedades modernas y la caída de sus instituciones representativas (la familia, la religión, etc.), pues no operan con la certeza o eficacia de antaño.

Empero, los desafíos actuales no pueden alejarnos de "(...) la importancia de las experiencias tempranas y su impacto en la psicopatología del adulto" (Romo \& Patiño, 2014, p.69). Pues estas, evidencian la necesidad de ocuparse de la protección y el cuidado de los procesos madurativoconstitucionales de la infancia. Fossa (2013) señala que "Las investigaciones en psicología evolutiva y los grandes teóricos del desarrollo concuerdan en que las experiencias tempranas son 


\section{Viana Ángela Bustos Arcón, Ana Rita Russo de Sánchez}

fundamentales en la constitución de la mente" (p.151). En el afán de ocuparse de las necesidades como sociedad, se ha olvidado la importancia de las bases del desarrollo del individuo, la infancia, pues no basta solo con atender la salud, educación, agua potable, sino, muy primordialmente el desarrollo emocional temprano, y con mayor rigor en casos de interferencias en el desarrollo. EI escenario colombiano ocupa un sinfín de dificultades afectivas y sociales en la infancia, las ciencias humanas parecen escasas frente a sus problemáticas, y en sus efectos, o sea, la salud mental: los trastornos mentales y las dificultades relacionales. Por lo que es urgente “(...) promover la recuperación física y psicológica y la reintegración social de todo niño víctima de: cualquier forma de abandono, explotación o abuso; tortura u otra forma de tratos o penas crueles, inhumanos o degradantes; o conflictos armados" (UNICEF, 1989, artículo 39, p.36). El desarrollo psicoafectivo es una prioridad, y las cifras en materia de salud mental en la infancia sugieren que las interferencias en el desarrollo impiden el curso esperado de lo biopsicosocial vital para la consolidación de las potencialidades humanas y la personalidad.

La psicología dinámica (Bayona, 2012) comprende que “(...) Las pautas básicas se establecen durante el proceso de maduración emocional, y los factores más influyentes son los que actúan al comienzo" (Winnicott, 1970a, pp.48-49), es decir, que ocupan un lugar determinante en el desarrollo psicoafectivo y en las raíces de la personalidad adulta. Más aún, se considera que "(...) la salud mental no depende solo de la herencia ni de acontecimientos fortuitos, sino que sus fundamentos se construyen en forma activa en la primera infancia (...)" (Winnicott, 1965, p.206). Por lo que la atención oportuna de las interferencias en el desarrollo, son vitales en la salud óptima durante la infancia (Phillips, 1988; Tamez, Ortega, Roa, Russo \& Galindo, 2006; Rangel, 2009).

Durante este tiempo diversas teorías de la salud mental en el ciclo vital de la vida han emergido. Pasando por Freud, Klein, Bowlby, Jung, Erikson, Piaget y Vygotsky, múltiples teorías (...) algunas de ellas (...) han dejadouna indeleble huella en el entendimiento actual de cómo el humano vive y experimenta la salud mental y cómo la pierde. (Romo \& Patiño, 2014, p.68)

Siendo que "El niño es el nivel más frágil de una estructura (...)" (Cohen, 2015, p.11), no puede ser deliberada su protección. En consecuencia, es urgente promover "La tendencia a la recuperación (...)" (Winnicott, 1963, p.85), ello implica, comprender, desarrollar y perfeccionar acciones, estrategias y programas en pro de la recuperación psicoafectiva como un modelo integrador de los procesos madurativo-constitucionales de la infancia en condiciones de interferencias en el desarrollo.

\subsection{Salud mental y Organización Mundial de la Salud- OMS}

La salud mental en Colombia y en el mundo se sustenta desde la Organización Mundial de la Salud (OMS) en un modelo biopsicosocial, y se comprende como “(...) un estado de bienestar en el que la persona materializa sus capacidades y es capaz de hacer frente al estrés normal de la vida, de trabajar de forma productiva y de contribuir al desarrollo de su comunidad" (OMS, 2013, p.42), por lo que todas las acciones en materia de salud mental “(...) implican la creación de condiciones individuales, sociales y ambientales que permitan el óptimo desarrollo psicológico y psicofisiológico" (OMS, 2004, p.17) de los individuos y su comunidad. La salud mental "[...] se asocia 


\section{Viana Ángela Bustos Arcón, Ana Rita Russo de Sánchez}

con cuatro concepciones de la salud mental: a) como ausencia de síntomas disfuncionales, b) como bienestar físico y emocional, c) como calidad de vida y d) como presencia de atributos individuales positivos" (Escobar \& Cova, 1997, citado por Palacio \& Martínez, 2007, p.116).

La salud mental es además “(...) una parte indivisible de la salud general, contribuye a las funciones de la sociedad y tiene un efecto sobre la productividad general" (OMS, 2004, p.23).

El bienestar físico, psicológico y social, que va más allá del esquema biomédico, donde abarca la esfera subjetiva y del comportamiento del ser humano. No es la ausencia de alteraciones y de enfermedad, sino un concepto positivo que implica distintos grados de vitalidad y funcionamiento adaptativo. (Oblitas, 2004, p.3)

La infancia es “(...) mucho más que el tiempo que transcurre entre el nacimiento y la edad adulta. Se refiere al estado y la condición de la vida de un niño: a la calidad de esos años" (UNICEF, 2004, p.1). Es más, "La infancia es una etapa crítica del desarrollo humano en la cual se siembran las semillas de la salud mental y el bienestar del futuro" (Benjet, 2009, p.234). Se refiere a los procesos madurativo-constitucionales vitales del desarrollo humano, y esto implica las condiciones necesarias para la salud mental. Es prioridad comprender las complejidades de la actualidad, y concentrar esfuerzos en atender los efectos de los desafíos contemporáneos, en especial en la población infantil.

(...) se reconoce que el ser humano nace con un alto grado de inmadurez; si bien es cierto que las pautas del desarrollo están dadas biológicamente, también lo es que se necesita de la relación madre-padre-ambiente para que el desarrollo pueda ocurrir, siendo la infancia el tiempo durante el cual se organizan los procesos emocionales y los elementos que sustentan la constitución de la personalidad. (Russo, 2014, p.18)

El desarrollo humano está dado también por el valor que representa la infancia para la sociedad, en la actualidad el niño/a ocupa un lugar privilegiado en materia de derechos, y esto ha permitido que año tras año, se generen nuevos compromisos nacionales e internacionales para su cuidado y protección.

La inscripción social de la significación del niño en la cultura occidental, remite a las representaciones colectivamente construidas en el transcurrir de los siglos que perfilan para cada época una imagen de niño a partir de la cual, las expectativas y los intercambios sociales entre adultos y niños logran un sentido particular que atraviesa las prácticas cotidianas entre ellos y proyecta una dialéctica de relaciones que tiene que ver con el futuro de cada sujeto y de cada sociedad. (López, 1999, p.1)

Es decir que, cuanto más se reconoce el valor transcendental de la infancia en la constitución de la sociedad, mayor son los esfuerzos por garantizar su pleno desarrollo. El interés fundamental es “(...) ejercer un impacto sobre los determinantes de la salud mental con el fin de aumentar la salud mental positiva" (OMS, 2004, p.17). Lamentablemente, la infancia resulta ser también foco de atropellos que impiden que los esfuerzos derivados de nuevas políticas para su protección se cumplan en propiedad. 


\section{Viana Ángela Bustos Arcón, Ana Rita Russo de Sánchez}

Las condiciones sociales juegan un papel importante en la salud mental de los individuos, en especial durante la infancia, pues la inmadurez de los niños y niñas los obliga a depender de los adultos, las instituciones y la sociedad en general (Freud, 1977; Serguéyevna, 2015). Se sabe que "La salud mental está influenciada no solo por factores internos de la persona (como la genética y la resiliencia personal) sino también de manera muy importante por el entorno" (Benjet, 2009, p.237). La inmadurez estructural de la infancia convierte a los niños y niñas, vulnerables a situaciones como la pobreza, el conflicto armado, la violencia, el maltrato, el abuso, etc., haciéndolos blanco de “(...) cualquier forma de abandono, explotación o abuso; tortura u otra forma de tratos o penas crueles, inhumanos o degradantes; o conflictos armados" (UNICEF, 1989, artículo 39, p.36), constituyéndose estas situaciones en interferencias en el desarrollo.

Una interferencia en el desarrollo deberá ser comprendida como toda aquella situación o evento externo que no haga parte de los conflictos esperados del desarrollo y que además adquiera el carácter de acontecimiento traumático, es decir que, “(...) se impone y nos aturulla (...)” (Cyrulnik, 2003, p.33). Pues sobrepasa todas las condiciones madurativo-constitucionales de los individuos, especialmente de los niños/as. Es decir, "Los menores de edad son en particular muy vulnerables a su entorno, ya que cuentan con pocos recursos o poder para cambiarlo" (Benjet, 2009, p.237), dado que los recursos emocionales y psíquicos se encuentran en desarrollo, y debido a que las interferencias en el desarrollo de las que han sido objeto, superan su capacidad para elaborar sus efectos. Por otro lado, cualquiera sea la interferencia en el desarrollo, las consecuencias podrían ser nefastas sino se atienden, consecuencias que pueden ir desde el aislamiento afectivo, agresividad o depresión, hasta alteraciones permanentes de la personalidad, y muy significativamente en todos los casos dificultades en el establecimiento de vínculos afectivos y relaciones interpersonales (Cyrulnik, 2003). Por lo que la atención de las interferencias en el desarrollo requiere ser integral, hace falta algo más que restituir los derechos de los niños/as, es preciso restituir el carácter emocional que sucumbe frente a los acontecimientos traumáticos, se requiere conceder estabilidad emocional y sentido a la realidad.

Se entiende que cuando los menores son expuestos a interferencias en el desarrollo, se interrumpe su curso esperado y como consecuencia se ve comprometida la salud mental. Por ejemplo, al producirse un abuso (en cualquiera de sus formas) los cambios comportamentales son evidentes, pero ¿qué de la naturaleza de los acontecimientos psíquicos que tal abuso representa en la infancia? Es allí, donde la interferencia en el desarrollo ocupa la prioridad para la atención integral. Los derechos pueden ser restituidos por medios legales, sociales y culturales, mientras que la condición emocional representada en tal interferencia deberá ser tratada desde su naturaleza, es decir, en su carácter emocional y relacional. En este caso es necesario “(...) promover la recuperación física y psicológica y la reintegración social de todo niño víctima (...)” (UNICEF, 1989, artículo 39, p.36). Y favorecer así la recuperación psicoafectiva en la infancia y disminuir los efectos negativos de la exposición a situaciones de vulnerabilidad.

Muy a menudo, en América Latina y el Caribe los jóvenes deben enfrentar un entorno que pone en riesgo su bienestar emocional, ya que muchos de ellos viven en la pobreza, trabajan en la calle, 


\section{Viana Ángela Bustos Arcón, Ana Rita Russo de Sánchez}

tienen embarazos tempranos y están expuestos a la violencia dentro y fuera de sus hogares. (Benjet, 2009, p.237)

E incluso son más notorios los esfuerzos por atender las problemáticas que enfrentan los niños, niñas y jóvenes en el mundo y en Colombia, a veces más por el fracaso aparente que por los buenos resultados.

En la actualidad puede notarse el aumento del maltrato infantil a pesar de las estrategias que se han implementado para la prevención; se han propuesto un sinnúmero de explicaciones y opiniones acerca del tema y sin embargo no se ha logrado el impacto deseado en las estadísticas. (OMS, 2013)

La salud mental en Latinoamérica como en el resto del mundo, posee dificultades operativas para atender los desafíos actuales. Pues, requiere robustecer las acciones dirigidas a atender las necesidades emocionales y sociales de la infancia, con el fin de seguir fortaleciendo las garantías de cuidado y protección (De la Fuente, 2014).

La oportunidad de aportar con una lectura profunda sobre las consecuencias clínicas de los individuos en un contexto que ha sido adverso al despliegue de las condiciones de su salud, respetando su singularidad y capacidad de crecimiento emocional, pero sobre todo en la atención de las condiciones humanas de la sociedad. (Bustos, 2016, p.2)

La salud mental “(...) debe ir más allá del conteo de frecuencias y de la descripción detallada de síndromes y trastornos en las etapas discretas de la vida" (Romo \& Patiño, 2014, p.68). Debe entenderse como el resultado de la confluencia de lo heredado y lo ambiental, y cuando esta relación se ve alterada requiere de nuevas condiciones internas y externas que permitan reestablecer "La tendencia a la recuperación (...)" (Winnicott, 1963, p.85) que los individuos poseen, dado que “(...) el propio proceso de desarrollo implica mayor plasticidad y por ende mayor adaptabilidad y receptividad a ser influenciado por él” (Benjet, 2009, p.237). O sea, que la inmadurez estructural durante la infancia, es también la condición necesaria para la recuperación psicoafectiva, pues los procesos madurativo-constitucionales inacabados operan siempre en favor de la integración, y ello constituye la salud mental.

\subsection{La salud mental como efecto de la integración del yo}

El desarrollo infantil, fue, es y será un tema siempre relevante y actualizado para la psicología, pues representa el principio de todas condiciones posibles de las raíces de las emociones, los afectos, las funciones mentales superiores, la maduración, etc. Es durante la infancia que lo primordial del desarrollo humano tiene lugar, y ello constituye una prioridad para su comprensión y estudio.

El desarrollo infantil integral suele definirse a través de los cambios que los niños atraviesan en términos físicos, cognitivos, emocionales y sociales que los habilitan a una vida autónoma y plena. Muchas de estas dimensiones son por todos reconocidas y solemos encontrar abundante literatura sobre la mayoría de estas (salud en primera infancia, desarrollo cognitivo, físico, entre otros). Sin embargo, es menos habitual contar con información relevante sobre un pilar del desarrollo infantil como lo es el desarrollo emocional. 


\section{Viana Ángela Bustos Arcón, Ana Rita Russo de Sánchez}

Aun así, el desarrollo infantil se nos presenta parcializado, y en ocasiones se favorecen las tendencias biológicas y en otras la importancia se centra en los factores ambientales, sobre lo que Anna Freud (1979) fue clara en sostener que “(...) nuestra tarea no consiste en aislar estos dos factores y en atribuir a cada uno un determinado campo de influencia, sino en trazar sus interacciones" (p.72). El desarrollo emocional será entonces el resultado de la relación entre lo innato y lo ambiental, o sea que la "(...) aparición de formas, de función y de conducta que son el resultado de intercambios entre el organismo, de una parte, y el medio interno y externo de otra" (Spitz, 1969, p.18). El despliegue del desarrollo emocional se determina por la incidencia de las relaciones entre el organismo y el ambiente.

El individuo nace en un estado de inmadurez y nodiferenciación entre el mundo interno y el mundo externo. Trae consigo una dotación genética que al integrarse a los procesos de maduración y desarrollo dará inicio a los precursores de los esquemas y representaciones del sí mismo y del mundo externo, integrando los componentes pulsional, cognitivo y afectivo. (Russo \& Mendoza, 2015, p.34)

Es preciso comprender que "El proceso madurativo-constitucional es universal, pero el desarrollo emocional concede a cada individuo las características particulares que posee" (Bustos, 2017, p.10). Esto quiere decir, que cualesquiera sean las condiciones de los individuos, el desarrollo tiene lugar en las particularidades de cada uno. Empero, el desarrollo en general y a lo largo de la vida se caracteriza por tener una orientación más o menos fija sobre la cual dirigir los procesos madurativo constitucional.

Estas líneas del desarrollo revelan los intentos del yo por enfrentar con éxito las situaciones de la vida, sin repliegues dolorosos y sin inhibición demasiado defensiva. Estas situaciones de la vida incluyen la separación de la madre, el nacimiento de un hermano o hermana, las enfermedades, la escuela, las relaciones con los pares, el juego, el surgimiento del interés sexual en la pubertad y, por supuesto, el amor. (Freud, 1962, p.34, citado por Sollod, Wilson \& Monte, 2009, p.140)

El resultado de los esfuerzos más o menos fijos del desarrollo son en efecto la consolidación de las raíces de la personalidad, o sea “(...) maduración, adaptación y estructuración...” (Freud, 1979, p.65). En definitiva, se sostiene que "La base de la salud mental es una personalidad bien integrada" (Klein, 1960, p.272). El desarrollo infantil es un esfuerzo constante por lograr la integración. Vital es que “(...) lo que sucede en las etapas tempranas del desarrollo de la personalidad (...) es integración, aplicable a casi todas las tareas evolutivas" (Winnicott, 1967a, p.35), por tanto, la salud mental está determinada por las características de integración que posea un individuo.

Ahora bien, la integración de las condiciones del desarrollo es la que permite establecer las “(...) generalidades del desarrollo y el modo particular del niño para afrontar cada una de las fases de la estructuración y desarrollo psíquico" (Russo \& Mendoza, 2015, p.36). O como bien afirmó Winnicott (1967a) cuando dice: "La integración conduce (...) al estado de unidad (...)" (p.35). O sea, todas las dimensiones del desarrollo se encuentran en una estrecha relación de tal forma que dependen unas de otras para que los procesos madurativo-constitucionales puedan suceder, y se pueda producir la integración. 


\section{Viana Ángela Bustos Arcón, Ana Rita Russo de Sánchez}

Los factores innatos, los factores ambientales y lo psíquico se ordena en función de lograr una unidad en el desarrollo, esta última es lo que representa la salud mental, pues "Cada fase del desarrollo contribuye de manera característica al desenvolvimiento de la personalidad" (Blos, 2011, p.35) y ordena una "(...) creciente diferenciación psíquica y una síntesis yoica de complejidad cada vez mayor y de un orden superior" (Blos, 2011, p.45), es así que, los “(...) aspectos más profundos del desarrollo emocional, tales como la riqueza de la personalidad, la fuerza del carácter y la capacidad de alcanzar una autoexpresión plena, libre y madura" (Winnicott, 1951, p.200), solo puede tener lugar desde la paulatina complejidad de los procesos madurativos, en especial cuando la consolidación del yo como instancia psíquica opera.

Pues en el yo, se ordenan las dimensiones físicas, cognitivas, afectivas y sociales, a partir de la satisfacción, la frustración y la realidad interna y externa, emergen los mecanismos de defensa, se produce la adaptación y estructuración psíquica. Se comprende que "El desarrollo y consolidación de la fortaleza yoica es el indicio básico de la salud" (Winnicott, 1963, p.86). De la capacidad del yo de hacer frente a los conflictos básicos o a la adversidad se evidencia la salud mental. Por ejemplo: "El niño no tiene emociones por sí mismo; la emoción solo tiene sentido en relación con una persona ya conocida" (Dolto, 2005, p.110). Con esto, se advierte que los afectos tienen sentido, son organizados e interpretados gracias a los procesos madurativo-constitucionales. Al crecer, las necesidades vitales son rápidamente transformadas en relaciones afectivas y dependiendo de esta relación se comprende el desarrollo mental.

Los momentos psicopatológicos o las patologías del desarrollo pueden hacer su aparición a lo largo del desarrollo por daños estructurales, carencias, fijaciones, regresiones, detención, interferencias o desviaciones en combinación con deficiencias, faltantes o sobresatisfacciones del ambiente psicoemocional inmediato (objeto primario) y/o el ambiente en el que el niño se desarrolla. (Russo \& Mendoza, 2015, p.36)

Curiosamente con frecuencia se asocia la salud mental con dimensiones positivas y un estado permanente de felicidad, cuando en realidad la salud mental es paradojal, porque ocupa al "(...) ser humano en tanto es, siente, actúa, se relaciona y medita" (Winnicott, 1965, p.201). Es decir, aunque el yo tenga la capacidad de ordenar y dar sentido a lo que le acontece, no significa que lo haga sin apremio, o que a través de lo que ordena no se experimente ansiedad. El destete, el complejo de Edipo, la castración, la rivalidad fraterna, entre otros conflictos constitucionales (que no se explican aquí), se corresponden con procesos complejos del yo para lidiar con la ansiedad que generan y que pertenecen al carácter madurativo de la infancia, sin que representen trastornos o problemas mentales, así como los afectos asociados a ellos como la rabia, la ambivalencia y el amor, no constituyen más que las expresiones propias de los conflictos vividos, por demás necesarios para el pleno desarrollo de la personalidad.

Como consecuencia, el mundo interno de la persona sana, aunque relacionado con el mundo externo o real, es personal y posee una vivacidad que le es propia. Las identificaciones introyectivas y proyectivas son incesantes. De ello se deduce que la pérdida y la mala suerte (y también la enfermedad, como ya lo he mencionado) pueden ser más terribles para las personas sanas que para 


\section{Viana Ángela Bustos Arcón, Ana Rita Russo de Sánchez}

las psicológicamente inmaduras o deformadas. La salud, debemos admitirlo, encierra sus propios riesgos. (Winnicott, 1967a, pp.38-39)

La salud mental, es integración, y la integración se desarrolla a partir de los conflictos evolutivos constitucionales, por lo que la capacidad de ordenamiento del yo no ocurre sin apuro, pues se necesita de una permanente adaptación del yo a las necesidades y demandas externas e internas, los mecanismos de defensa evolucionan de acuerdo a los conflictos estructurales y la experiencia externa. Un individuo sano, comienza con un niño sano. La salud mental se organiza tempranamente, y desde con lo anímico, y es el yo, la instancia psíquica que esta personifica.

\section{MÉTODO}

El presente artículo tiene por objetivo comprender cómo el concepto de salud mental se relaciona con las nociones de interferencias en el desarrollo y recuperación psicoafectiva. Se deriva además de la tesis doctoral denominada Validación de un programa de recuperación psicoafectiva en interferencias en el desarrollo. A continuación, se relacionan las especificaciones de su realización.

\subsection{Diseño}

Se enmarca en la investigación cualitativa, y en particular en la investigación documental. Siendo un estudio metódico, sistemático y organizado (Uribe, 2013) de textos, artículos y documentos sobre un tema (Packer, 2013; Strauss \& Corbin, 2012; Bernal, 2010). El método corresponde a “(...) una abstracción científica (...) una labor interpretativa (...) según su naturaleza analítica (...)" (Uribe, 2013, p.198) y comporta “(...) un proceso no matemático de interpretación, realizado con el propósito de descubrir conceptos y relaciones en los datos (...) luego organizarlos en un esquema explicativo teórico" (Strauss \& Corbin, 2012, p.12). Por tanto, la revisión documental aquí realizada, conduce a explicaciones teóricas acerca de la salud mental y la integración.

\subsection{Participantes e instrumentos}

Los instrumentos comprenden la organización de las fuentes de consulta, que han sido seleccionadas de acuerdo con la teoría psicodinámica, y su delimitación conceptual se refiere a: salud mental e integración. Los textos aquí seleccionados son de carácter bibliográfico, cuyo “(...) análisis de la información escrita sobre un determinado tema, con el propósito de establecer relaciones, diferencias, etapas, posturas (...) del conocimiento respecto al tema objeto de estudio" (Bernal, 2010, p.111). Así mismo, los textos escogidos comprenden niveles comprensivos e interpretativos, acerca de salud mental e integración únicamente en la teoría psicodinámica (Winnicott, 1969; 1970b).

Dado que es una investigación de carácter documental, no implica la participación de sujetos en el desarrollo de sus aproximaciones conceptuales ni en sus resultados. Sin embargo, su alcance sí podría implicar la participación de sujetos en otras modalidades y diseños de investigación.

Por otra parte, se someten a la comunidad científica los resultados aquí encontrados y bajo las disposiciones editoriales de la revista. 


\section{Viana Ángela Bustos Arcón, Ana Rita Russo de Sánchez}

\subsection{Procedimientoy análisis de datos}

Recolección de información: Los estudiantes de los grupos que quedaron en la muestra diligenciaron el instrumento AF5 bajo la supervisión de las docentes investigadoras, y firmaron el consentimiento informado. Variables utilizadas en la investigación: Programa, semestre, género, puntajes académico-laboral, social, emocional, familiar y físico.

El procedimiento y análisis de los datos se realizó de acuerdo con las características de la investigación cualitativa, en la modalidad de investigación documental y en un carácter intra-teórico acudiendo a "(...) escritos (artículos, informes, planificadores, etc.), entrevista, observaciones, entre otros" (Valbuena, 2013, p.214). Para ello se realizó una jerarquización de las fuentes correspondiente a dos objetivos primordiales, primero comprender los conceptos de salud mental e integración, y en segundo lugar generar explicaciones de las condiciones de salud mental en la infancia. Los autores aquí revisados pertenecen a una línea de primer nivel ocupada por Anna Freud, Melanie Klein y Donald Winnicott. El segundo nivel es ocupado por autores recientes que han derivado estudios acerca de la temática, y en tercer nivel, todos aquellos autores o textos, que pertenecen o no a la teoría psicodinámica o que de forma indirecta abordan la temática revisada, pero que favorecen la discusión y análisis de la salud mental en la infancia.

Operativamente el procedimiento de la selección de textos se estableció así: a. Definición del objetivo de acuerdo con la fase exploratoria de la tesis doctoral Validación de un programa de recuperación psicoafectiva en interferencias en el desarrollo, la teoría psicodinámica y autores pertenecientes al concepto de desarrollo emocional en la infancia y la salud mental. b. Búsqueda, selección y jerarquización de los textos, documentos, libros, artículos, etc., de acuerdo con las categorías de salud mental e integración. c. Finalmente se establecen relaciones teóricas entre la salud mental y la integración durante la infancia.

El análisis de los datos, es decir, de los documentos, textos, artículos, etc., revisados “(...) tienen por objeto elaborar y procesar datos relevantes sobre las condiciones mismas en que se han producido aquellos textos, o sobre las condiciones que puedan darse para su empleo posterior" (Piñuel, 2002, p.2). Opera en el orden comprensivo-explicativo de la salud mental como efecto de la integración en la infancia, a partir de la selección de los documentos, jerarquización de los textos, la organización categorial, el análisis categorial y las aproximaciones comprensivo-explicativas obtenidas.

\section{RESULTADOS}

La salud mental (López, 2012) es un tema urgente de atender, ocupa no solo a la disciplina psicológica y la psiquiatría sino también a la clase política y económica, pues representa las condiciones del desarrollo de los estados y la capacidad para el despliegue de las potencialidades humanas. La prioridad de la salud mental es cuidar los factores que la promueven y atender las necesidades que surgen cuando se pierde. Es vital comprender que "La salud es un estado y al mismo tiempo, un proceso dinámico cambiante" (Mebarak, De Castro \& Salamanca, 2009, p.3). Lo anterior cobra valor si entendemos que la salud mental es el efecto del desarrollo, puesto que, al 


\section{Viana Ángela Bustos Arcón, Ana Rita Russo de Sánchez}

complejizarse los procesos internos y externos del individuo, se dinamizan sus capacidades biopsicosociales y se consolida la personalidad.

En otras palabras, podemos decir que el crecimiento y el desarrollo psicológico son el resultado de una compleja relación que en principio se da entre la díada madre-hijo, para luego pasar a la tríada madre-padre hijo, en interacción con el medio ambiente y la dotación constitucional del niño. (Russo \& Mendoza, 2015, p.35)

El cuidado, protección y atención de la salud mental en la infancia es un paso a favor de las transformaciones posibles que tienen lugar en la adolescencia, o sea, “(...) la etapa de la vida durante la cual el individuo busca establecer su identidad adulta, apoyándose en las primeras relaciones objétales parentales internalizadas" (Aberastury \& Knobel, 1998, p.15). Esto permite considerar que la salud mental del adulto es un reflejo de la salud mental infantil.

La adolescencia se “(...) caracteriza por la proliferación de rasgos y por la construcción de múltiples versiones del yo, acomodándose a una ecología social cada vez más compleja (puede actuar de forma distinta según la situación)" (Cantón, Cortes \& Cantón, 2011, p.171), se afirma de esta manera que los procesos básicos de la salud mental se determinan en parte por los procesos madurativoconstitucionales y la consolidación del yo. Así pues, la "Salud Mental sería la capacidad potencial de realizarnos en nuestra propia vida, y esta capacidad no nos es dada de una vez por todas, sino que debemos conquistarla permanentemente a través del ciclo vital" (Cortese, 2004, p.106). O sea que es el resultado de la conquista permanente de los conflictos de la vida, y ocupa el terreno a todos los individuos y de la sociedad en general.

El desarrollo infantil esperado es la garantía para la salud mental, el logro principal del desarrollo es laintegración de las dimensiones biopsicosociales en la infancia que facilitan la "(...) maduración, adaptación y estructuración (...)” (Freud, 1979, p.65). Aunque “(...) en la cuestión del desarrollo aparecen inevitablemente recortes, precisamente porque lo que se desarrolla son las funciones y no el sujeto" (Jerusalinsky et al., 2000, p.18), es necesario comprender que la salud mental es sinónimo de integración, y no ocurre de forma positiva o grata, sino bajo los avatares del desarrollo, los conflictos psíquicos, la complejidad de las relaciones afectivas y el contexto social.

Por ello “(...) una característica de la salud es que (...) nunca deja de desarrollarse (...)” (Winnicott, 1967a, p.35). Y que contrasta con el hecho de que "Por definición y debido a los procesos de maduración, la apetencia por completar el desarrollo es muchísimo más marcada durante la inmadurez que en ninguna otra etapa posterior de la vida" (Freud, 1979, p.29). Es posible sostener que la condición esencial de la salud mental es el cambio, la transformación y su dinamismo, so pena del desarrollo mismo. Es decir, cuanto más complejo es el proceso madurativo-constitucional, más compleja resulta la salud mental. La responsabilidad de la disciplina psicológica en cuidar y atender el problema de la salud mental es trascendental en la medida en que su objetivo asistencial y científico radica en comprender el comportamiento humano, las dimensiones que lo constituyen, y además favorecer el despliegue de las potencialidades humanas.

El para qué de la psicología es la transformación, el cambio. Esto nos plantea la necesidad de explicitar la dirección y sentido de este cambio. La psicología dinámica plantea que salud mental no 


\section{Viana Ángela Bustos Arcón, Ana Rita Russo de Sánchez}

significa ausencia de síntomas ni adaptación acrítica a la norma, sino, la capacidad de lo humano para reconocer su procesoy cuestionándolo modificar su estructura y desarrollo. (Ávila \& De Pablos, 1978)

Es preciso entonces, atender la salud mental y "(...) conocer las tensiones que son inherentes a la vida y los medios que se emplean para hacerles frente" (Winnicott, 1961a, p.20). Las líneas de desarrollo guían el reconocimiento de las complejidades estructurales de los procesos madurativoconstitucionales y su ocurrencia facilita la asistencia al desarrollo psicoafectivo. Este reconocimiento, permitiría en el caso de las interferencias en el desarrollo establecer no solo las interrupciones y efectos de su acontecer, sino además determinar las necesidades asistenciales y ocuparse de la recuperación psicoafectiva.

Es posible prever semejante catástrofe evolutiva cuando no se hace nada, cuando los estereotipos culturales estigmatizan a los niños, cuando se dice que son monstruosos, que no tienen arreglo, que serán toda la vida unos retrasados, unos gérmenes de delincuencia, cuando el Estado no construye ninguna institución que les dinamice, cuando las familias exhaustas o mal constituidas impiden que se teja todo vínculo afectivo, o cuando los adultos responsables, al no creer en la posibilidad de recuperar a esos niños, no disponen a su alrededor ningún tutor de resiliencia. (Cyrulnik, 2003, p.49)

En el caso de interferencias en el desarrollo, es más complejo pues implica anticiparse a los efectos de estas, o sea, los síntomas clínicos y las graves consecuencias en el desarrollo biopsicosocial del momento y la personalidad posterior. Por esto, en el caso de las interferencias en el desarrollo, cuanto más temprana sea la intervención psicológica, mayores efectos positivos se podrían obtener, en beneficio de los procesos madurativo-constitucionales, la integración y la salud mental en la infancia.

\section{DISCUSIÓN}

Hasta el día de hoy, la salud mental se piensa en función a la enfermedad mental o los trastornos emocionales, como una suerte de problemas estructurales per se, y no como el resultado de los procesos madurativo-constitucionales. Es decir, un trastorno mental no es el efecto de ecuaciones generales o psiquiátricas, sino resultado del proceso particular de desarrollo y crecimiento de un individuo. En otras palabras, la salud mental es un problema del desarrollo humano, y este tiene su inicio en la infancia, por lo que la salud mental es un problema del desarrollo infantil.

La salud mental se comprende como un logro de la adultez, y no como el resultado de los paulatinos alcances del ciclo vital, es decir, que la salud mental y la mala salud se entienden de acuerdo con los síntomas y no en sus causas. De acuerdo con esta afirmación, el desarrollo psicoafectivo es el asidero de lo que se conoce como salud mental, las condiciones biopsicosociales y emocionales tienen un valor importante de forma integral, siempre y cuando se reconozca que "El afecto es una necesidad vital (...)” (Cyrulnik, 2003, p.34). Pues ningún individuo es una isla, ninguna sociedad está hecha de un individuo, ninguna cultura se forma sin vínculos. 


\section{Viana Ángela Bustos Arcón, Ana Rita Russo de Sánchez}

Los países del mundo hacen esfuerzos inmensos por atender la infancia, sin embargo, priorizan factores económicos por encima de las necesidades emocionales vitales de los individuos, el desarrollo emocional de los niños/as y la crianza emocional es urgente si se comprende la salud mental como un efecto del desarrollo psicoafectivo, o sea, como resultado de los numerosos las necesidades de los ciudadanos. En el caso de la infancia, en condiciones de interferencias en el desarrollo, previamente mencionadas, la recuperación psicoafectiva emerge como una opción viable a las problemáticas que deben afrontar miles de niños en Colombia.

Los derechos del niño constituyen un reto para crear y sostener condiciones necesarias y suficientes para que todos los niños y niñas, sin excepción, puedan disfrutar a plenitud de buenas condiciones de salud, estar bien nutridos, con un óptimo desarrollo psicosocial, y respetados por y protegidos por sus padres, adultos y (...) por las leyes de cada país. (Amar, Madariaga \& Macías, 2014, p.29)

La cuestión a no desatender, se refiere al compromiso disciplinar de la psicología, y en particular de la psicología clínica de establecer las condiciones necesarias para la protección y cuidado de la salud mental temprana, y no solo en atender los efectos de la mala salud, es preciso reiterar que la salud mental comprende los esfuerzos vitales y madurativo-constitucionales por lograr la integración de las dimensiones totales del desarrollo, y como resultado de la superación de los conflictos propios de los momentos evolutivos y los avatares que implica cada uno.

El campo de la psicología clínica integra ciencia, teoría y práctica para entender, predecir y aliviar el desequilibrio, la invalidez y la incomodidad; también promueve la adaptación humana, el ajuste y el desarrollo personal.

La psicología clínica está enfocada en los aspectos intelectuales, emocionales, biológicos, psicológicos, sociales y del comportamiento humano que funcionan a través de la existencia en las diferentes culturas, y en todos los niveles socioeconómicos. (Cullari, 2001, p2) Resulta obvio pensar que "iEs tan fácil perturbar el desarrollo emocional...!" (Winnicott, 1951, p.199). Si consideramos las condiciones biopsicosociales necesarias para el óptimo despliegue del desarrollo y sus potencialidades, pues cualquier desajuste podría provocar las más dañinas consecuencias en la formación de la personalidad y la salud mental.

No es viable desconocer que "El maltrato infantil es tenido hoy como una problemática de alta incidencia, que prevalece como reto a la mirada clínica de nuestros tiempos (Mass, 2011, p.5). El Código de Infancia y Adolescencia (2006) al promover la protección y cuidado de los derechos niños, niñas y adolescentes, también convoca a la rama jurídica, a la rama científica y a sociedad en general a pensar las condiciones biopsicosociales de los individuos en el país.

Se entiende por maltrato infantil toda forma de perjuicio, castigo, humillación o abuso físico o psicológico, descuido, omisión o trato negligente, malos tratos o explotación sexual, incluidos los actos sexuales abusivos y la violación y en general toda forma de violencia o agresión sobre el niño, la niña o el adolescente por parte de sus padres, representantes legales o cualquier otra persona. (Art. 18, p.344) No es concebible el desarrollo sin la dimensión contextual, pues “(...) el crecimiento emocional del individuo, incluye la evolución de su capacidad para relacionarse con las personas y con el ambiente en general" (Winnicott, 1961b, p.120), y esto solo puede lograrse en la medida en 


\section{Viana Ángela Bustos Arcón, Ana Rita Russo de Sánchez}

que la unidad del desarrollo haya tenido lugar, no solo por el contexto, sino por los efectos que las relaciones producen en la vida anímica y la integración. Es claro que "Un niño no es fragmentable, es un ser total cuya mejoría corporal se asocia al progreso del lenguaje, y cuya inteligencia se alía con la afectividad" (Cyrulnik, 2003, p.51). Por tanto, en el caso de las interferencias en el desarrollo, esta unidad se ve amenazada y todos los ámbitos de su desarrollo se ven alterados.

El desafío que los fenómenos humanos han representado para los intereses investigativos, puede reflejarse en la realización de estudios que conciben al sujeto no solo desde el sentido de un desarrollo único, sino además como el producto de una historia familiar; esto se genera, gracias a la motivación en propuestas comprensivas sobre lo subjetivo y su modo de articulación cultural. (Mass, 2013, p.36)

Ahora bien, en materia de recuperación psicoafectiva en Colombia y en el mundo no existe desarrollo científico en el tema, tampoco programas, estrategias o proyectos dirigidos a las interferencias en el desarrollo de orientación dinámica. El programa de recuperación El Retorno de la Alegría implementado en Bolivia, Honduras, el Salvador, Perú y República Dominicana (ajustado a las condiciones específicas de los países), únicamente se ocupa de atender niños/as o adolescentes en emergencias naturales (UNICEF, 2007). En Colombia, su implementación se realizó con Nidya Quiroz (1999) denominado Recuperación psicoafectiva de niños afectados por desastres y conflicto armado. En ambas condiciones, se utilizan las estrategias lúdicas, comprendiendo el momento evolutivo y el apoyo de familiares y profesionales para favorecer el ajuste psicológico en las condiciones mencionadas.

Empero, según el Instituto Colombiano de Bienestar Familiar-ICBF han ingresado a la institución hasta el 2013 un total de 16.457 niños, niñas y adolescentes víctimas de maltrato infantil, por otro lado 1.709.995 niños, niñas y adolescentes fueron víctimas de desplazamientos forzado (ICBF, 2013, p.1) y 11.126 niños han sido víctimas de trabajo infantil. Es decir, que son muchas más las interferencias en el desarrollo, las que aquejan a la infancia permanentemente, en comparación con el conflicto armado o los desastres naturales, o sea, que existe una deuda pendiente con la infancia en condición de vulnerabilidad en Colombia.

(...) objetivo último sería diseñar programas tanto de prevención de la enfermedad como de promoción y mantenimiento de la salud, mediante un conocimiento contextualizado de nuestra realidad psicológica (en sus aspectos afectivos y cognoscitivos) y socioculturales, para que, basados en dicho conocimiento y comprensión, se realicen programas orientados al fortalecimiento de una red de servicios de salud y al desarrollo de estilos de vida sanos y coherentes a las necesidades concretas de nuestro entorno inmediato que, a su vez, también permitan reducir el impacto negativo de nuestra problemática psicosocial sobre las distintas poblaciones, comunidades, grupos, organizaciones y familias. (Mebarak, De Castro \& Salamanca, 2009, p.5)

Para las ciencias sociales y para las ciencias médicas es necesario favorecer todas las condiciones posibles en beneficio de la salud mental de niños, jóvenes y adultos.

Para trabajar sobre el problema o necesidad que precisa análisis e intervención, tenemos que definirlo en términos de conductas problema, establecer indicadores para evaluarlo y analizar la 


\section{Viana Ángela Bustos Arcón, Ana Rita Russo de Sánchez}

pertinencia de esos indicadores (...) Definir operativamente el problema es describirlo en términos de las variables relevantes que lo componen, que nos permitan su medida, manejo y/o su tratamiento (Rodríguez, Cañadas, García \& Mira, 2007, p.54)

Se ha dicho que la salud mental es un efecto del, desarrollo, es porque se refiere a la más alta complejidad de la integración de los procesos madurativo-constitucionales y representa el carácter de unidad que es el individuo, una unidad paradojal, pues comprende los conflictos propios del ser humano, sus relaciones, deseos y frustraciones. La salud mental, así como el desarrollo está caracterizada por su dinamismo y la permanente necesidad de adaptación que convierte al yo en la instancia psíquica vital de la salud mental. El yo es la integración y la unidad, no sin premura. Pues su esencia es la dinámica de las demandas internas y externas, la realidad y la satisfacción. Por tanto, todos los esfuerzos en materia de salud deben centrarse en dotar a la comunidad y a los individuos, padres, niños y niñas, en comprender la paradoja estructural que es la salud mental, su dimensión biopsicosocial y dotar al yo de una capacidad de discernimiento y autoobservación.

Entonces, ¿qué sucede cuando en la temprana infancia ocurre una brusca interrupción del mundo que rodea al niño? ¿Qué sucede cuando esta brusca interrupción no es seguida de reparación? ¿Qué sucede cuando esta experiencia traumática temprana se caracteriza por un ambiente permanente de muerte y de violencia? ¿Qué sucede cuando los padres ya nunca más serán los mismos de los primeros momentos de la vida? (Gómez, 2013, p.154)

Los acontecimientos traumáticos al ocurrir por fuera de los conflictos esperados del desarrollo deberán ser atendidos, pero para ello se requiere de una comprensión vital de la complejidad de la salud mental, su intervención no debe sostenerse solo en un discurso de derechos y síntomas, deberá centrarse en las causas y construirse a partir de la complejidad vital de la salud mental, para ello es necesario contener un referente teórico que permita dirigir las acciones clínicas. La psicología clínica opera por fuera del consultorio, y desde sus principios es posible construir estrategias de orientación analítica si: Se comprende la relación existente entre la salud mental y el ciclo vital temprano, las interferencias en el desarrollo y la mala salud, y se reconocen los conflictos madurativos como un sistema organizador de la vida emocional. Cyrulnik (2003) afirma que "No hay duda que este tipo de reconstrucción transita muy cerca de la angustia y el agotamiento, ¿pero quién ha dicho que la resiliencia fuese un camino fácil?" (p.59). En definitiva, en el caso de las interferencias en el desarrollo, sus efectos son seguros, pero para que estos sean menos dramáticos o impidan la realización individual se requiere de: Establecer vínculos afectivos estables (familia y entorno), Dar sentido, Resignificar y Empoderar.

La discusión aquí consagrada acerca de la salud mental como efecto de la integración no se considera terminada, más bien ofrece la oportunidad de revalidar los hallazgos teóricos aquí encontrados y transformarlos en acciones, programas y proyectos que continúen promoviendo la salud mental en la infancia, junto a la familia y la comunidad en general. Es preciso resolver el problema de la salud mental cuando esta ha sido interrumpida a causa de situaciones como el trauma, y conducir a los afectados a la recuperación psicoafectiva en condiciones como el conflicto armado, el desplazamiento, el abuso o el maltrato. Adicionalmente, urge el desarrollo de investigaciones centradas en la recuperación psicoafectiva e interferencias en el desarrollo. Empero, 
la psicología dinámica y la comprensión de la integración del yo y su relación con la salud mental conduciría a una renovación en las tradicionales formas de atender la salud mental en la infancia, y dirigirse a una visión emocional y evolutiva.

Nota de Autores

Artículo derivado del proyecto de tesis doctoral: Validación de un programa de recuperación psicoafectiva en interferencias en el desarrollo. Universidad del Norte

\section{REFERENCIAS}

Aberastury, A. \& Knobel, M. (1998). La adolescencia anormal. Un enfoque psicoanalítico. México: Editorial Paidós. Recuperado de: https://psiqueunah.files.wordpress.com/2014/09/melita-aberastury-laadolescencia-normal.pdf

Amar, J., Madariaga, C. \& Macías A. (2014). Infancia, familia y derechos humanos. Barranquilla: Editorial Universidad del Norte. Recuperado de: https://books.google.com.co/books?id=uLBCDwAAQBAJ\&pg=PP6\&dq=Infancia,+familia+y+derech os+humanos.+Barranquilla:+Editorial+Universidad+del+Norte.\&hl=es-

419\&sa=X\&ved=0ahUKEwjRp9_hjYDaAhURY6wKHZ9LAPAQuwUILDAA\#v=onepage\&q=Infancia\%2C \%20familia\%20y\%20derechos\%20humanos.\%20Barranquilla\%3A\%20Editorial\%20Universidad\%20 del\%20Norte.\&f=false

Ávila, A. \& De Pablos, P. (28 de abril de 1978). La psicología científica de orientación dinámica. Diario El País. Recuperado de: https://elpais.com/diario/1978/04/28/sociedad/262562421_850215.html

Bayona, M. (2012). La noción de matriz relacional y sus implicaciones para el ejercicio clínico de la psicología dinámica. Revista Psicología desde el Caribe, 29(3), 686-706. Recuperado de: http://rcientificas.uninorte.edu.co/index.php/psicologia/article/viewFile/3321/9162

Benjet, C. (2009). Salud mental de la niñez y la adolescencia en América Latina y el Caribe. En Epidemiología de los trastornos mentales en América Latina y el Caribe. Organización Panamericana de la Salud. Oficina Regional de la Organización Mundial de la Salud. Recuperado de: http://apps.who.int/iris/ bitstream/10665/166275/1/9789275316320.pdf?ua=1

Bernal, C. (2010). Metodología de la investigación. Editorial Pearson. Recuperado de: https://books.google.com.co/books?id=h4X_eFai59oC\&printsec=frontcover\&dq=Bernal,+C.+(2010 ).+Metodolog\%C3\%ADa+de+la+investigaci\%C3\%B3n.+Editorial+Pearson\&hl=es419\&sa=X\&ved=0ahUKEwiosZqOjoDaAhVPZKwKHWalDwcQ6AEIJZAA\#v=onepage\&q\&f=false 
Blos, P. (2011). Los comienzos de la adolescencia. Buenos Aires: Amorrortu Editores. Recuperado de: http://www.academia.edu/19745591/Blos_Peter_1979_La_transicion_adolescente

Bustos, V. (2016). Psicoanálisis, postconflicto y salud mental. Revista Psicogente, 19(36), 202-203. Recuperado de: http://revistas.unisimon.edu.co/index.php/psicogente/article/view/1361/1346

Cantón Duarte, J., Cortés Arboleda, M. \& Cantón Cortés, D. (2011). Desarrollo socioafectivo y de la personalidad. Madrid: Alianza Editorial.

Campo, L.A. (2014). El desarrollo del autoconcepto en niños y niñas y su relación con la interacción social en la infancia. Revista Psicogente, 17(31),67-79. Doi: 10.17081/psico.17.31.422

Cyrulnik, B. (2003). El murmullo de los fantasmas. Barcelona: Editorial Gedisa. Código de Infancia y Adolescencia (2006). Ley 1098. Recuperado de: https://www.oas.org/dil/esp/ Codigo_de_la_Infancia_y_la_Adolescencia_Colombia.pdf

Cohen, S. (2015). La niñez cautiva. Salud mental infantil y juvenil. México: Fondo de Cultura Económica. Recuperado de: https://www.fce.com.ar/ar/libros/detalles.aspx?IDL=7709

Cortese, E. (2004). Los conceptos de normalidad-anormalidad, salud-enfermedad. En Psicología médica salud mental (pp. 101-109). Buenos Aires: Nobuko.

Cullari, S. (2001). Fundamentos de Psicología Clínica. México: Pearson. Recuperado de: https://www.researchgate.net/publication/274836451_Fundamentos_de_Psicologia_Clinica_Span ish_Edition

De la Fuente, R. (2014). Introducción a la salud mental. En Salud mental y medicina psicológica (pp. 2-5). México: McGraw-Hill. Recuperado de: https://accessmedicina. mhmedical. com/content. aspx?bookid=1440\&sectionid=94746599

Dolto, F. (2005). La dificultad de vivir. Familia y sentimientos 1. Barcelona: Editorial Paidós. Duro, E. (2012). Prólogo. En Desarrollo emocional. Clave para la primera infancia. UNICEF \& KALEIDOS. Recuperado de: https://www.unicef.org/ecuador/Desarrollo_emocional_0a3_simples.pdf

Fossa, P. (2013). La desorganización psíquica en el desarrollo infantil. Revista GEPU, 4(1), 150-159. Recuperado de: https://revistadepsicologiagepu.es.tl/La-Desorganizaci\%F3n-Ps\%EDquica-en-elDesarrollo-Infantil.htm

Freud, A. (1977). Psicoanálisis del niño. Argentina: Ediciones Hormé. Recuperado de: http://www.academia.edu/5559063/Freud_Anna_-_Psicoanálisis_del_Niño_-_Ed._Paidós

Freud, A. (1979). Normalidad y patología en la niñez. Argentina: Editorial Paidós Psicología Profunda. Recuperado de: https://es.scribd.com/doc/225244697/Freud-Anna-Normalidad-Y-Patologia-En-LaNinez-pdf

Gómez, E. (2013). Trauma relacional temprano. Chile: Ediciones Universidad Alberto Hurtado. Recuperado de: 
https://books.google.com.co/books?id=MZcBCgAAQBAJ\&printsec=frontcover\&source=gbs_ge_su

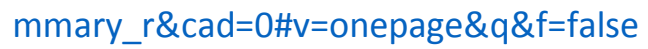

Greenspan, S. (1992). Infancy and Early childhood: The practice of clinical assessment and intervention with emotional and developmental challenges. Madison: International University Press. Recuperado de: http://psycnet.apa.org/record/1992-97299-000

ICBF (2013). Maltrato infantil. Dirección de Protección ICBF. Recuperado de: http://www.icbf.gov.co/ portal/page/portal/Descargas1/Prensa1/ColombiaSinMaltatoInfantil_180313.pdf

Jerusalinsky, A., Arias, M., Brandao, P., Bruckman, S., Coriat, E., Coriat, L. \&Tkach, C. (2000). Psicoanálisis en problemas del desarrollo infantil. Una clínica transdisciplinaria. Buenos Aires: Editorial Nueva Visión. Recuperado de: https://www.researchgate.net/publication/31647735_Psicoanalisis_en_problemas_del_desarrollo _infantil_una_clinica_transdisciplinaria_A_Jerusalinsky

Klein, M. (1960). Sobre la salud mental. En Envidia y gratitud. Y otros trabajos (pp. 272-278). Argentina: Editorial Paidós. Recuperado de: https://es.scribd.com/document/349705579/01-Klein-M-1960Sobre-La-Salud-Mental

López, Y. (1999). De la inocencia del niño a la sexualidad infantil. Affectio Societatis. Revista Electrónica del Departamento de Psicoanálisis, 2(4). Recuperado de: http://aprendeenlinea.udea.edu.co/revistas/index.php/affectiosocietatis/article/view/5410/4762

López, L. (2012). Atención en salud mental en el sector público y privado. Factores que influyen en la eficacia del tratamiento. Revista Psicogente, 15(27), 198-206. Recuperado de: http://revistas.unisimon.edu.co/index.php/psicogente/article/view/1909

Martínez, M., Robles, C., Utria, L. \& Amar, J. (2014). Legitimación de la violencia en la infancia: un abordaje desde el enfoque ecológico de Bronfenbrenner. Revista Psicología desde el Caribe, 31(1), 133-160. Recuperado de: http://rcientificas.uninorte.edu.co/index.php/psicologia/article/viewFile/4930/9119

Mass, L. (2011). Familia contemporánea y maltrato infantil. Una lectura desde la teoría psicoanalítica. Alemania: Editorial Académica Española. Recuperado de: https://www.researchgate.net/profile/Leonardo_Mass_Torres/publication/317698813_Familia_y _maltrato_infantil_Una_Revision_Teorica_en_torno_a_la_Clinica_Contemporanea_Articulo_prod ucto_de_una_revision_teorica/links/59498828a6fdcc3e17fc6ce4/Familia-y-maltrato-infantil-UnaRevision-Teorica-en-torno-a-la-Clinica-Contemporanea-Articulo-producto-de-una-revisionteorica.pdf

Mass, L. (2013). Familia y maltrato infantil: Una revisión teórica en torno a la clínica contemporánea. Revista CES, 4(2), 35-43. Recuperado de: https://www.researchgate.net/profile/Leonardo_Mass_Torres/publication/317698813_Familia_y _maltrato_infantil_Una_Revision_Teorica_en_torno_a_la_Clinica_Contemporanea_Articulo_prod ucto_de_una_revision_teorica/links/59498828a6fdcc3e17fc6ce4/Familia-y-maltrato-infantil-Una- 


\section{Viana Ángela Bustos Arcón, Ana Rita Russo de Sánchez}

Revision-Teorica-en-torno-a-la-Clinica-Contemporanea-Articulo-producto-de-una-revisionteorica.pdf

Mass, L. (2014). El sujeto y la estética corporal en la sociedad contemporánea (algunas relaciones teóricas con el capitalismo y plus de gozar). Revista Psicogente, 17(31), 155-162. Recuperado de: http:// publicaciones.unisimonbolivar.edu.co:82/rdigital/psicogente/index.php/psicogente/article/ viewFile/428/393m

Mebarak, M., De Castro, A. \& Salamanca, M. (2009). Salud mental: un abordaje desde la perspectiva actual de la psicología de la salud. Revista Psicología desde el Caribe, (23). Recuperado de: http://www.scielo.org.co/pdf/psdc/n23/n23a06.pdf

Minsalud \& Colciencias (2015). Encuesta Nacional en Salud Mental. Tomo I. Bogotá: Javegraf. Recuperado de: $\quad$ https://www.minsalud.gov.co/sites/rid/Lists/BibliotecaDigital/RIDE/DE/presentacionencuesta-nacional-salud-mental-2015.pdf

Oblitas, L. (2004). Psicología de la salud y calidad de vida. México: Thompson. Recuperado de: http://www.unife.edu.pe/pub/revpsicologia/psicologiasalud.pdf

OMS (2004). Prevención de los Trastornos Mentales. Intervenciones efectivas y opciones de políticas. Informe compendiado. Un Informe del Departamento de Salud Mental y Abuso de Sustancias de la Organización Mundial de la Salud. Recuperado de: http://www.who.int/mental_health/evidence/Prevention_of_mental_disorders_spanish_version. pdf

OMS (2013). Plan de acción para la salud mental 2013- 2020. Recuperado de: http://www.who.int/mental_health/publications/action_plan/es/

Packer, M. (2013). La ciencia de la investigación cualitativa. Bogotá: Ediciones Uniandes. Recuperado de: http://repositorio.uniandes.edu.co/xmlui/handle/1992/15522

Palacio, J. \& Martínez, Y. (2007). Relación del rendimiento académico con la salud mental en jóvenes universitarios. Revista Psicogente, 10(18), 113-128. Recuperado de: http://revistas.unisimon.edu.co/index.php/psicogente/article/view/1556

Piñuel, J. (2002). Epistemología, metodología y técnicas del análisis del contenido. Revista Estudios de Sociolingüística, $\quad 3(1), \quad 1-42 . \quad$ Recuperado: https://www.researchgate.net/publication/267797356_Epistemologia_metodologia_y_tecnicas_d el_analisis_de_contenido

Phillips, A. (1988). Winnicott. Buenos Aires: Bruner. Recuperado de: https://books.google.com.co/books?id=NFJxuGNHPNIC\&printsec=frontcover\&source=gbs_ge_su mmary_r\&cad $=0 \# v=$ onepage $\& q \& f=$ false

Quiroz, N. (1999). Programa Recuperación psicoafectiva de niños afectados por desastres y conflicto armado. Recuperado de: http://www.unicef.org/republicadominicana/cartilla_metodologia.pdf 


\section{Viana Ángela Bustos Arcón, Ana Rita Russo de Sánchez}

Rangel, M. (2009). Psicoterapia infantil. Un enfoque psicoanalítico. México: Editorial Trillas. Recuperado de: https://www.magisterio.com.co/libro/psicoterapia-infantil-un-enfoque-psicoanalitico

Rodríguez, J., Cañadas, I., García, J. \& Mira, J. (2007). El diseño de un programa de intervención. En Intervención Psicosocial (pp. 47-73). Madrid: Pearson/ Prentice Hall.

Romo, F. \& Patiño, L. (2014). Ciclo vital y salud mental. 201 cia de Implementación en Colombia1997-2014. En Experiencias Significativas en Psicología y Salud Mental. Bogotá: Colpsic.

Russo, A. \& Mendoza, A. (2015). La entrevista psicológica inicial con el niño y sus padres y el proceso psicodiagnóstico. En Temas en psicología clínica (pp. 28-59). Barranquilla: Universidad del Norte Editorial. Recuperado de: https://books.google.com.co/books?id=kEpb5f82XgC\&pg=PA4\&lpg=PA4\&dq=La+entrevista+psicol\%C3\%B3gica+inicial+con+el+ni\%C3\%B1o+y+su $\mathrm{s}+$ padres+y+el+proceso+psicodiagn\%C3\%B3stico\&source=bl\&ots=xOg4Z2qPpY\&sig=L5GtD23jaEW Ke39azeQ2mZH1jll\&hl=es419\&sa=X\&ved=0ahUKEwjx9aiHiYDaAhULDKwKHRwTCbcQ6AEIQDAC\#v=onepage\&q=La\%20entre vista\%20psicol\%C3\%B3gica\%20inicial\%20con\%20el\%20ni\%C3\%B10\%20y\%20sus\%20padres\%20y\% 20el\%20proceso\%20psicodiagn\%C3\%B3stico\&f=false

Serguéyevna, V. (2015). Psicología evolutiva. España: Machado Nuevo Aprendizaje. Recuperado de: https://www.iberlibro.com/PSICOLOGÍA-EVOLUTIVA-Valeria-Serguéyevna-MújinaANTONIO/15815612529/bd

Sollod R., Wilson J. \& Monte, C. (2009). Teorías de la personalidad. Debajo de la máscara. Octava edición. McGraw-Hill. Recuperado de: http://booksmedicos.org/teorias-de-la-personalidad-debajo-de-lamascara-8a-edicion/

Spitz, R. (1969). El primer año de vida del niño. México: Fondo de Cultura Económica. Recuperado de: https://www.elfondoenlinea.com/Detalle.aspx?ctit=007041R

Strauss, A. \& Corbin, J. (2012). Bases de la investigación cualitativa. Técnicas y procedimientos para desarrollar la teoría fundamentada. Colombia: Editorial Universidad de Antioquia. Recuperado de: https://diversidadlocal.files.wordpress.com/2012/09/bases-investigacion-cualitativa.pdf

Tamez, R., Ortega S., Roa, A., Russo A. \& Galindo, J. (2006). Educación y desarrollo psicoafectivo. Barranquilla: Ediciones Uninorte. Recuperado de: https://books.google.com.co/books?id=3M4ilHgpNgC\&pg=PR5\&lpg=PR5\&dq=).+Educaci\%C3\%B3n+y+desarrollo+psicoafectivo.+Barranquilla:+E diciones+Uninorte.\&source=bl\&ots=MaRPDmtc-r\&sig=Twx5svMJTY941yvdjSdD3ECKKXI\&hl=es419\&sa=X\&ved=0ahUKEwi5iY2I4ezZAhVopVkKHVWjB1kQ6AEITTAD\#v=onepage\&q=).\%20Educaci \%C3\%B3n\%20y\%20desarrollo\%20psicoafectivo.\%20Barranquilla\%3A\%20Ediciones\%20Uninorte.\&f $=$ false

UNICEF (1989). Convención sobre los derechos del niño. Recuperado de: https://www.unicef.org/argentina/spanish/7.-Convencionsobrelosderechos.pdf

UNICEF (2004). Estado mundial de la infancia 2005. La infancia amenazada. Recuperado de: https://www. unicef.org/spanish/sowc05/sowc05_sp.pdf 
UNICEF (2007). Programa: El retorno de la alegría. Recuperado de: http://www.unicef.org/republicadominicana/MANUAL_TERAPIAS_Y_JUEGOS.pdf

Uribe, J. (2013). La investigación documental y el estado del arte como estrategias de investigación en ciencias sociales. En La investigación en Ciencias Sociales: Estrategias de investigación (pp. 197-212). Bogotá: Universidad Piloto de Colombia.

Valbuena, E. (2013). El análisis del contenido: De lo manifiesto a lo oculto. En La investigación en Ciencias Sociales: Estrategias de investigación (pp. 213-224). Bogotá: Universidad Piloto de Colombia.

Winnicott, D. (1951). Las bases de la salud mental. En: Deprivación y delincuencia (pp. 197-200). Buenos Aires: Editorial Paidós.

Winnicott, D. (1961a). Psicoanálisis y ciencia: camigos o parientes? En El hogar, nuestro punto de partida. Ensayos de un psicoanalista (pp. 17-23). Buenos Aires: Editorial Paidós.

Winnicott, D. (1961b). Variedades de psicoterapia. En El hogar, nuestro punto de partida. Ensayos de un psicoanalista (pp. 117-129). Buenos Aires: Editorial Paidós.

Winnicott, D. (1963). El valor de la depresión. En El hogar, nuestro punto de partida. Ensayos de un psicoanalista (pp. 84-93). Buenos Aires: Editorial Paidós.

Winnicott, D. (1965). El niño en el grupo familiar. En El hogar, nuestro punto de partida. Ensayos de un psicoanalista (pp. 149-163). Buenos Aires: Editorial Paidós.

Winnicott, D. (1967a). El concepto de individuo sano. En El hogar, nuestro punto de partida. Ensayos de un psicoanalista (pp. 27-47). Buenos Aires: Editorial Paidós.

Winnicott, D. (1969). De la pédiatrie á la psychanalyse. Paris: Payot.

Winnicott, D. (1970a). Vivir creativamente. En El hogar, nuestro punto de partida. Ensayos de un psicoanalista (pp. 48-65). Buenos Aires: Editorial Paidós. 